\title{
The potential role of mesenchymal stem cell therapy for intervertebral disc degeneration: a critical overview
}

\author{
Frank L. Acosta Jr., M.D., Jeffrey Lotz, Ph.D., and Christopher P. Ames, M.D. \\ Departments of Neurological and Orthopaedic Surgery, University of California, San Francisco, \\ California
}

\begin{abstract}
Low-back pain is the most common health problem for men and women between 20 and 50 years of age, resulting in 13 million doctor visits in the US annually, with significant costs to society in terms of lost time from work and direct and indirect medical expenses. Although the exact origin of most cases of low-back pain remains unknown, it is understood that degenerative damage to the intervertebral disc (IVD) plays a central role in the pathogenic mechanism leading to this disorder. Current treatment modalities for disc-related back pain (selective nerve root blocks, surgical discectomy and fusion) are costly procedures aimed only at alleviating symptoms. Consequently, there is growing interest in the development of novel technologies to repair or regenerate the degenerated IVD. Recently, mesenchymal stem cells (MSCs) have been found to possess the capacity to differentiate into nucleus pulposus-like cells capable of synthesizing a physiological, proteoglycan-rich extracellular matrix characteristic of healthy IVDs. In this article, the authors review the use of MSCs for repopulation of the degenerating IVD. Although important obstacles to the survival and proliferation of stem cells within the degenerating disc need to be overcome, the potential for MSC therapy to slow or reverse the degenerative process remains substantial.
\end{abstract}

\section{KEY WORDS • back pain • intervertebral disc • disc degeneration • stem cell • tissue engineering $\bullet$ disc regeneration}

\section{OVERVIEW}

Back pain is a cause of significant morbidity in all Western industrialized societies. ${ }^{42,43}$ It is estimated that up to $80 \%$ of the population in these societies will experience some form of back pain over their lifetime, with approximately $10 \%$ of sufferers becoming chronically disabled. ${ }^{42-44}$ Not only does this result in a significant amount of physical and emotional distress for patients and their families, but it is also an enormous economic burden. The societal costs for medical treatment, lost time from work, and disability benefits are on the order of $\$ 30$ billion annually in the US alone, ${ }^{2,23}$ and $£ 12$ billion per year in the United Kingdom..$^{25}$

Although there are numerous causes of back pain, it is clear that this disorder is strongly associated with degeneration of the IVD. ${ }^{5,20,22}$ Moreover, IVD degeneration is thought to contribute to spinal arthritis, radiculopathy, and myelopathy. ${ }^{33}$ Instead of focusing on repairing or reversing damage to the IVD, current treatment strategies, including nonsurgical (physical therapy, nerve root blocks) and surgical ones (disc excision and arthrodesis) effect only symp-

Abbreviations used in this paper: $\mathrm{CD}=$ cluster of differentiation; $\mathrm{ECM}=$ extracellular matrix; IL = interleukin; IVD = intervertebral disc; $\mathrm{MSC}=$ mesenchymal stem cell; $\mathrm{NP}=$ nucleus pulposus; $\mathrm{TGF} \beta=$ transforming growth factor $-\beta ; 3 \mathrm{D}=$ three-dimensional. tomatic relief and may actually accelerate the same degenerative process at adjacent levels. Thus, degeneration of the IVD is currently regarded as an irreversible phenomenon with no effective treatment. Nevertheless, recent advancements in the understanding of tissue repair mechanisms and stem cell biology have led to the identification of novel tissue engineering-based treatment strategies to regenerate or repopulate the diseased IVD with stem cells. ${ }^{8,11}$. 13,18,31-35

In this paper we comment on the ability of MSCs to slow or reverse the degenerative process, on potential obstacles to stem cell survival and proliferation within the degenerating disc, and we conclude with future directions for research aimed at overcoming these barriers.

\section{Molecular Aspects of IVD Degeneration}

Alterations in Disc Collagen Content. Collagen content, including the relative amounts of various collagen subtypes, is crucial to proper disc function. ${ }^{9}$ Young, healthy discs exhibit active matrix formation and turnover of Type II collagen, whereas aging discs demonstrate decreased matrix production and Type II collagen denaturation. ${ }^{3}$ Degenerating discs are marked by decreased Type II procollagen formation, increased Type II collagen denaturation, and Type I collagen synthesis. Degenerating discs also demon- 
strate a decrease in Type IX and an increase in Type X collagen, which may be a marker of active matrix repair.,28 Free radical-induced modifications (N-[carboxymethyl]lysine) to disc collagen have also been noted within degenerating IVDs, possibly indicating that oxidative stresses play an active role in the degenerative process. ${ }^{28}$

Alterations in Disc Proteoglycan and Water Content. Degenerating discs possess a relative increase in decorin and biglycan and a relative decrease in the 4-sulfated chondroitin sulfate component of large proteoglycans. ${ }^{19} \mathrm{An}$ increase in fibromodulin has been documented by immunoblot analysis of degenerating discs. ${ }^{41}$ Because there is a close interplay between proteoglycans and collagens of the disc ECM (fibromodulin, decorin, and lumican bind to Type I and Type II collagen, for example), ${ }^{15,16}$ changes in the content and function of one of these molecules can cause dramatic alterations in the other.

\section{Degradation of the ECM}

Liu, et al., ${ }^{21}$ found that pro-MMP-3 (also known as prostromelysin), which is secreted by cultured human discs, may contribute to age-related changes when activated within the disc ECM. Other proteolytic enzymes, including cathepsins, have been implicated in age-related changes to the disc and vertebral endplate. ${ }^{4,27}$

\section{Rationale for Cell-Based Approaches to Restore the IVD}

Currently, both nonsurgical and surgical therapies for degenerative disc disease target only symptomatic relief without addressing the underlying degenerative process. The concept of biological disc repair has grown in recent years because of improved understanding of the cellular and molecular events involved in disc aging and degeneration. ${ }^{10}$ Realization that disc degeneration is a cell-driven process has raised the possibility of manipulating cellular content and behavior toward a beneficial outcome. Such manipulation is necessary because the IVD has a limited vascularity and capacity for self-repair.

One approach is to signal existing disc cells in situ to secrete increased quantities of proteoglycan in attempts to increase swelling pressure. Growth factors TGF $\beta$, osteogenic protein-1, fibroblast growth factor, growth and differentiation factor-5, or bone morphogenetic protein-2, among others, have either been injected directly ${ }^{46}$ or their production in disc cells has been stimulated with gene therapy. ${ }^{29}$ Although promising results have been reported after use of these techniques, the relative acellularity of degenerated discs in humans raises concerns that the patient's own disc cells may be present in insufficient numbers to mount a therapeutic repair response. Therefore, the introduction of cells capable of surviving within the IVD and producing appropriate matrix is an important component of bioengineered regeneration.

Cell therapy seeks to restore a critical population of disc cells that will synthesize appropriate matrix in attempts to recover biomechanical properties. This approach was first attempted by performing transplantation of autologous NP tissue into denucleated rat discs, where it was shown to slow the progression of degeneration..$^{30}$ More recently, autologous disc cell implantation was proven to be technically feasible in sand rats, an animal model of age-related spon- taneous degeneration. ${ }^{14}$ This later study demonstrated the isolation and in vitro expansion of anular cells, their incorporation into a collagen carrier, and their reinsertion into adjacent discs in the same animals. Nevertheless, the effect of implanted cells on the degenerated state was not evaluated.

A commonality in each of these approaches is the use of disc chondrocytes as the cell type for regeneration. Ultimately, however, this cell source has practical limitations in the clinical setting because of graft procurement and harvest site morbidity. For example, harvesting the patient's own cells requires damage to an adjacent disc, likely inducing degeneration in that level. Also, the relative acellularity of disc tissue will require culture expansion, which is a slow process for disc cells. Furthermore, the cells acquired from one level to treat another will be approximately the same age and potentially limited as to the extent to which they may develop a therapeutic repair response. By contrast, adult MSCs are easily procured by bone marrow aspiration, readily expanded in culture, and may be more capable of adapting successfully to the environment of the IVD, and therefore of achieving a differentiated state appropriate for long-term matrix synthesis.

\section{Use of MSCs to Regenerate the NP}

The MSCs, or human bone marrow stromal stem cells, are nonhematopoietic bone marrow-derived pluripotent progenitor cells with the ability to generate cartilage, bone, muscle, tendon, ligament, and fat (Fig. 1). ${ }^{32}$ These cells exist postnatally, are uncommitted precursor cells, and have extensive renewal potential. They are identified by cell surface markers such as CD44, CD71, and CD166, but do not express hematopoietic lineage markers. ${ }^{6,33}$ They are also characterized by a particular pattern of cytokine production, including IL-6, IL-7, IL-8, IL-11, IL-12, and granulocyte colony-stimulating factor. ${ }^{24}$ The use of MSCs to restore the IVD eliminates the drawbacks associated with the use of autogenous disc cells, including limited supply, donor site morbidity, and limited regenerative capacity. Mesenchymal stem cells have indeed been shown to be capable of restoring damaged disc tissue in animal models. ${ }^{34}$ The differentiation of MSCs is governed largely by their surrounding environment. In vitro conditions that promote differentiation into a chondrogenic phenotype depend first on the presence of specific growth factors, and second on a 3D construct of cells to promote cell-cell interactions (Fig. 2).

\section{Differentiation of MSCs Into NP-Like Cells}

It is known that MSCs can differentiate into chondrocyte-like cells within the proper environment. ${ }^{45}$ Because NP cells and chondrocytes have many common features, it is reasonable to assume that MSCs also give rise to NP cells in an environment similar to the IVD (that is, in conditions of low oxygen tension and high stress). In a recent in vitro study, Risbud, et al., ${ }^{32}$ analyzed the ability of rat MSCs to differentiate into NP-like cells. Results from this study demonstrated that in the presence of hypoxia and TGF $\beta_{1}$, cultured MSCs express a phenotype consistent with NP cells via mitogen-activated protein kinase signaling pathways. Hypoxia caused an upregulation of matrix metalloproteinase-2, Types II and IX collagen, and aggrecan expression, whereas TGF- $\beta$ treatment increased collagen Type II and aggrecan gene expression. Moreover, these 


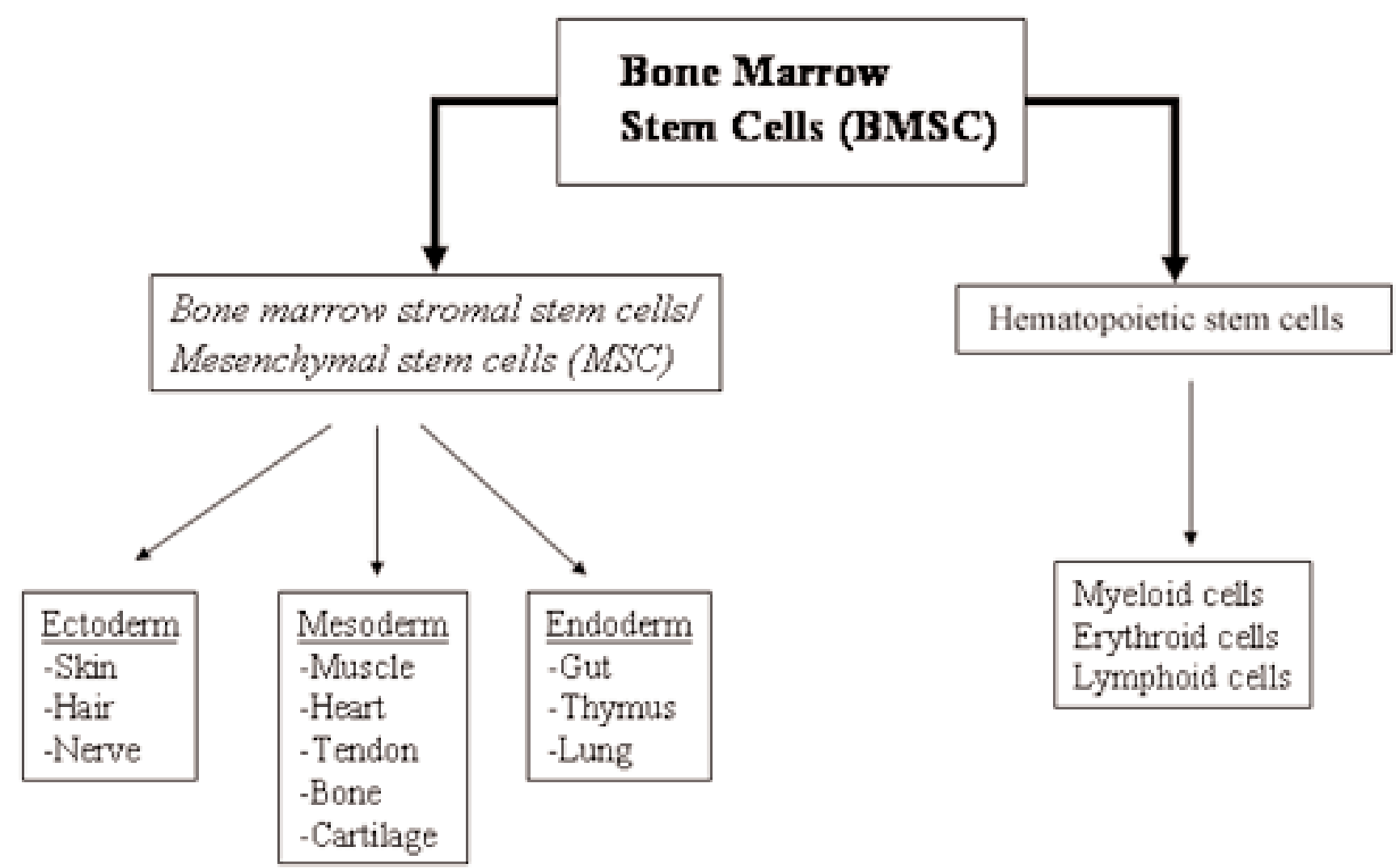

Fig. 1. Chart showing that bone marrow stem cells produce both hematopoietic and nonhematopoietic (mesenchymal) stem cells. The MSCs give rise to various tissues, including bone, cartilage, and tendon.

cells were demonstrated to be biosynthetically active by Alcian blue staining of the ECM. The authors concluded that MSC can be driven (by hypoxia in the presence of TGF- $\beta$ ) to commit to a lineage of NP-like cells that can potentially be used to repopulate the degenerating IVD.

The role of hypoxia in maintaining the NP cell phenotype has also been documented by others. ${ }^{33,36}$ Steck, et al., ${ }^{39}$ studied MSCs derived from human bone marrow samples. In this study they found that, in the presence of $\mathrm{TGF}_{3}$, dexamethasone, and ascorbate, MSC derived from bone marrow differentiated in spheroid culture toward a chondrogenic lineage. After TGFß-mediated differentiation, MSC spheroids were positive for collagen Type II, aggrecan, decorin, and fibromodulin at levels closer to those found in IVD tissue than to hyaline articular cartilage. The authors concluded that human bone marrow-derived MSC can be committed to adopt a gene expression profile more similar to IVD tissue than to native joint cartilage. It seems apparent, therefore, that TGF- $\beta$ and low oxygen tension are crucial elements in driving MSC to differentiate into NPlike cells.

\section{Use of 3D Scaffolds for MSC Culture}

The suitability of cultured MSCs for use in tissue engineering of the IVD depends not only on their commitment to an NP lineage, but also on their 3D organization with an NP-like phenotype. Similar to articular chondrocytes, a 3D culture environment (for example, micromass or alginate gel as opposed to monolayer culture) is necessary to maintain the disc cell phenotype in vitro. The pellet system is the one most commonly used to produce cells similar to chondrocyte condensations in vivo. Disc cells grown in monolayer assume a fibroblast-like phenotype. ${ }^{13}$ In a 3D environment, however, disc cells become rounded, form colonies, and exhibit greater proliferation and proteoglycan synthesis (Fig. 3).12,13 Various in vitro culture techniques, including complex 3D gels ${ }^{7,26,32}$ and degradable polymer scaffolds ${ }^{17}$ have been developed with the goal of providing a sustainable frame on which MSC can proliferate. Hyaluronic acid, collagen, and chitosan have been used as scaffold materials to provide immobilization of MSC in a 3D framework that maintains their NP-like phenotype..$^{31,40}$ Chitosan gel and fibrin glue have been used in cross-linkable polymeric preparations to entrap cells. ${ }^{37}$ These $3 \mathrm{D}$ in vitro culture techniques allow for stable cell anchorage and permit independent production of intercellular matrix, cytokines, and bioactive molecules. ${ }^{33}$

\section{Survival and Function of MSCs in the IVD}

Studies of the viability of MSCs after injection into the IVD have shed light on the feasibility of repopulating the degenerative NP with cells committed to the NP phenotype. In an in vivo model, Crevensten, et al., ${ }^{8}$ found an initial decrease in numbers of MSCs labeled with fluorescence 14 days after injection into rat coccygeal discs. Twenty-eight days postinjection, however, cell counts returned to the ini- 


\section{Bone marrow}

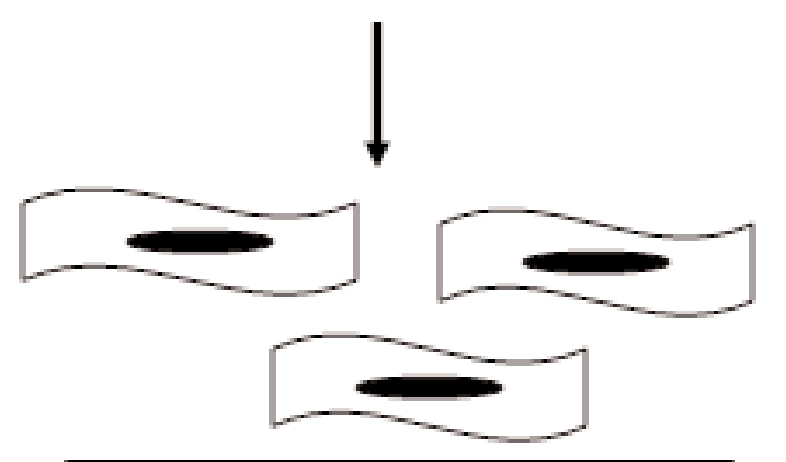

Mesenchymal stem cells
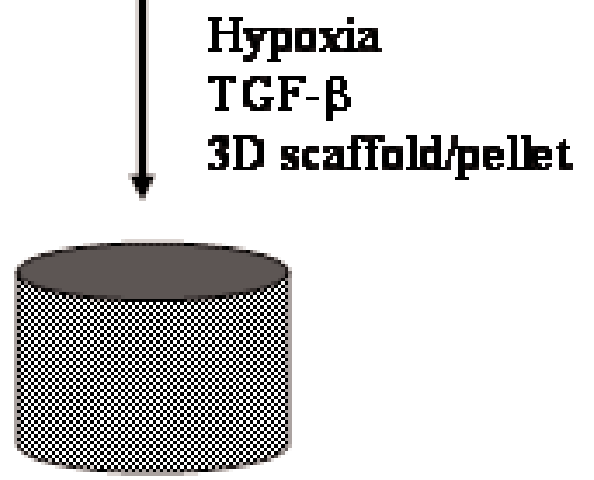

\section{Nucleus pulposus-like cells}

Fig. 2. Chart illustrating how bone marrow aspirates of MSCs are induced to differentiate into a chondrogenic/NP-like phenotype with certain growth factors and 3D culture conditions.

tial number of injected cells, with $100 \%$ viability. These authors also observed a trend of increased disc height compared with the control group, suggesting an increase in matrix synthesis. The authors concluded that MSC can maintain viability and proliferate within the rat IVD.

Zhang, et al. ${ }^{48}$ injected MSCs into rabbit IVDs to study their in vivo synthetic function. Transplanted MSCs were identified within the discs at 1,3 , and 6 months after injection. Reverse transcription-polymerase chain reaction and enzyme-linked immunosorbent assays were used to quantify MSC products. These authors found that the amount of proteoglycan and Type II collagen messenger RNA and protein were significantly increased in rabbit discs injected with MSCs compared with control discs.

Sakai, et al., ${ }^{34}$ induced premature disc degeneration in New Zealand white rabbits after aspiration of the NP. Injured discs transplanted with MSCs showed restoration of disc space, with persistence or reappearance of the NP. These phenomena were not observed in the control group.
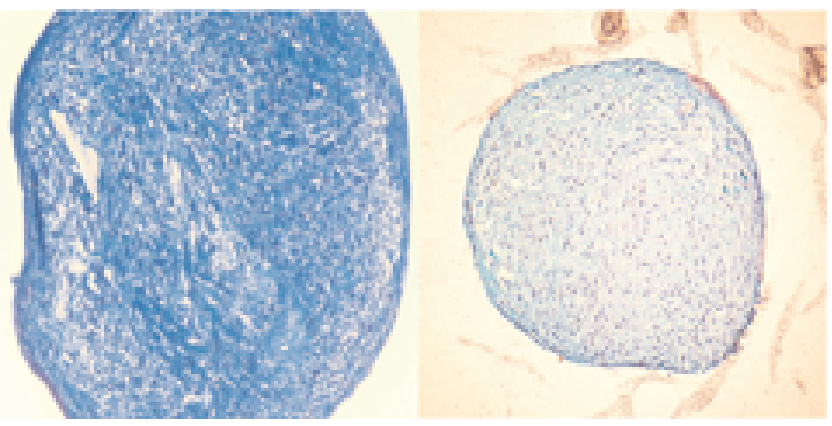

Fig. 3. Photomicrographs of sections of a human MSC pellet after 3 weeks of culture. The sections were stained with Alcian blue (left, original magnification $\times 20)$ and safranin O (right, original magnification $\times 10$ ), and demonstrate proteoglycan staining indicative of chondrogenic differentiation.

Moreover, proteoglycan synthesis in discs transplanted with MSC was similar to that in normal, uninjured discs. The authors postulated that regeneration of the IVD after MSC transplantation may be due to differentiation into NP cells or promoting regeneration in residual native NP cells. In any case, these in vitro and in vivo studies have shown that MSCs are capable of surviving and proliferating within the IVD and can potentially restore its normal structure and function.

\section{Activation of NP Cells by MSCs}

In addition to repopulating the IVD by differentiating into NP-like cells, MSCs may also prove effective in activating native NP cells to increase production of ECM, thus restoring disc height, swelling pressure, and normal biomechanical properties. Yamamoto, et al., ${ }^{47}$ found that cell proliferation, DNA synthesis, and proteoglycan production were significantly increased in NP cells cocultured with MSCs and grown with direct cell-to-cell contact. An increase in the expression of various growth factors known to activate the biochemical properties of the NP was also observed. Thus, activation and reimplantation of NP cells obtained during discectomy, for example, and then cocultured with MSC may be a useful source of native cells to restore the degenerated IVD.

\section{Limitations and Future Directions}

Transplantation of MSCs to humans has been effective for tissues such as cartilage and myocardium. ${ }^{38,45}$ Although MSCs are easily procured by bone marrow aspiration and hold significant potential to repopulate the degenerated NP, it is unclear whether endplate permeability in degenerated discs is sufficient to support increases in cell density and metabolism (because poor nutrition may have led to the degeneration in the first place). Furthermore, low pH, oxidative stresses, and inflammatory mediators present within the degenerated IVD may alter the behavioral and synthetic properties of transplanted MSCs. Thus, research focused on revealing the relationship between vertebral endplate permeability, disc nutrition, and disc cell health is central to understanding the feasibility of MSC regeneration of the IVD. Last, the biomechanical properties of the degenerated IVD, including distribution of mechanical loads and hydro- 
static pressures, might alter the properties of transplanted MSCs or prevent them from fully differentiating into functional, disc-like cells. Moreover, the properties of the carrier medium may be altered in the biomechanical environment of the degenerated IVD. Current research is focused on determining whether MSCs can be made to maintain a disc-like phenotype under such conditions. The results of these investigations will serve as guides for tissue engineering approaches to disc repair and will help establish whether disc repair strategies in humans will ultimately be limited by inherent nutritional deficiencies and alterations in biomechanical properties caused by pathological conditions in the endplate.

Future studies are required to determine the following: 1) the optimal cell type and $\mathrm{pH}$ level for transplantation; 2) the type of carrier medium for transplantation; 3 ) the behavior of MSCs in vivo; and 4) factors that may induce MSCs to differentiate into either NP-like cells or anular cells. Ultimately, human trials will be necessary to determine the safety, feasibility, and ultimate efficacy (both radiographically and clinically confirmed; that is, pain relief) of MSC transplantation.

\section{CONCLUSIONS}

Currently available modalities for the treatment of IVD degeneration aim only at alleviating symptoms of back pain, radiculopathy, and/or myelopathy, and fail to address the underlying disease process. Advancements in understanding the cellular basis of IVD degeneration and stem cell biology have led to significant progress in the field of tissue engineering to repair, reverse, or regenerate diseased tissue. As uncommitted precursor cells, MSCs have the potential to differentiate into NP-like cells with the ability to proliferate and repopulate the degenerated IVD directly, activate native NP cells, or deliver gene products of interest. Research is required to determine the optimal cell type and growth conditions for transplantation. Moreover, the behavior of MSCs in the in vivo environment of the degenerated disc has yet to be determined. The feasibility of MSC regeneration of the IVD depends on answering these questions. Although it is currently unavailable, MSC therapy to regenerate the IVD may prove to be an effective treatment strategy for patients with back pain related to degenerative disc disease. By addressing the underlying cellular abnormalities leading to degeneration of the IVD, MSC therapy may allow patients with back pain to avoid extensive and morbidity-producing fusion procedures, permitting an earlier return to normal function and productivity, and leading to significant economic savings to society in general.

\section{References}

1. Aigner T, Gresk-otter KR, Fairbank JC, et al: Variation with age in the pattern of type $\mathrm{X}$ collagen expression in normal and scoliotic human intervertebral discs. Calcif Tissue Int 63:263-268, 1998

2. Andersson GB: Epidemiology of low back pain. Acta Orthop Scand Suppl 281:28-31, 1998

3. Antoniou J, Steffen T, Nelson F, et al: The human lumbar intervertebral disc: evidence for changes in the biosynthesis and denaturation of the extracellular matrix with growth, maturation, ageing, and degeneration. J Clin Invest 98:996-1003, 1996

4. Ariga K, Yonenobu K, Nakase T, et al: Localization of cathep- sins $\mathrm{D}, \mathrm{K}$, and $\mathrm{L}$ in degenerated human intervertebral discs. Spine 26:2666-2672, 2001

5. Benneker LM, Heini PF, Alini M, et al: 2004 Young Investigator Award Winner: vertebral endplate marrow contact channel occlusions and intervertebral disc degeneration. Spine 30: 167-173, 2005

6. Campagnoli C, Roberts IA, Kumar S, et al: Identification of mesenchymal stem/progenitor cells in human first-trimester fetal blood, liver, and bone marrow. Blood 98:2396-2402, 2001

7. Chiba K, Andersson GB, Masuda K, et al: A new culture system to study the metabolism of the intervertebral disc in vitro. Spine 23:1821-1828, 1998

8. Crevensten G, Walsh AJ, Ananthakrishnan D, et al: Intervertebral disc cell therapy for regeneration: mesenchymal stem cell implantation in rat intervertebral discs. Ann Biomed Eng 32: 430-434, 2004

9. Eyre DR: Collagens of the disc, in Ghosh P (ed): The Biology of the Intervertebral Disc. Boca Raton, FL: CRC Press Inc, 1988, Vol 1, pp 171-188

10. Freemont AJ, Watkins A, Le Maitre C, et al: Current understanding of cellular and molecular events in intervertebral disc degeneration: implications for therapy. J Pathol 196:374-379, 2002

11. Gruber HE, Hanley EN Jr: Biologic strategies for the therapy of intervertebral disc degeneration. Expert Opin Biol Ther 3: 1209-1214, 2003

12. Gruber HE, Hanley EN Jr: Human disc cells in monolayer vs 3D culture: cell shape, division and matrix formation. BMC Musculoskelet Disord 1:1, 2000

13. Gruber HE, Hanley EN Jr: Recent advances in disc cell biology. Spine 28:186-193, 2003

14. Gruber HE, Johnson TL, Leslie K, et al: Autologous intervertebral disc cell implantation: a model using Psammomys obesus, the sand rat. Spine 27:1626-1633, 2002

15. Hedbom E, Heinegard D: Binding of fibromodulin and decorin to separate sites on fibrillar collagens. J Biol Chem 268: 27307-27312, 1993

16. Hedbom E, Heinegard D: Interaction of a 59-kDa connective tissue matrix protein with collagen I and collagen II. J Biol Chem 264:6898-6905, 1989

17. Honda M, Yada T, Ueda M, et al: Cartilage formation by cultured chondrocytes in a new scaffold made of poly(L-lactideepsilon-caprolactone) sponge. J Oral Maxillofac Surg 58: 767-775, 2000

18. Hunter CJ, Matyas JR, Duncan NA: The notochordal cell in the nucleus pulposus: a review in the context of tissue engineering. Tissue Eng 9:667-677, 2003

19. Inkinen RI, Lammi MJ, Lehmonen S, et al: Relative increase of biglycan and decorin and altered chondroitin sulfate epitopes in the degenerating human intervertebral disc. J Rheumatol 25: 506-514, 1998

20. Kurunlahti M, Tervonen O, Vanharanta H, et al: Association of atherosclerosis with low back pain and the degree of disc degeneration. Spine 24:2080-2084, 1999

21. Liu J, Roughley PJ, Mort JS: Identification of human intervertebral disc stromelysin and its involvement in matrix degradation. J Orthop Res 9:568-575, 1991

22. Luoma K, Riihimaki H, Luukkonen R, et al: Low back pain in relation to lumbar disc degeneration. Spine 25:487-492, 2000

23. Maetzel A, Li L: The economic burden of low back pain: a review of studies published between 1996 and 2001. Best Pract Res Clin Rheumatol 16:23-30, 2002

24. Majumdar MK, Thiede MA, Mosca JD, et al: Phenotypic and functional comparison of cultures of marrow-derived mesenchymal stem cells (MSCs) and stromal cells. J Cell Physiol 176:57-66, 1998

25. Maniadakis N, Gray A: The economic burden of back pain in the UK. Pain 84:95-103, 2000

26. Martin I, Suetterlin R, Baschong W, et al: Enhanced cartilage 
tissue engineering by sequential exposure of chondrocytes to FGF-2 during 2D expansion and BMP-2 during 3D cultivation. J Cell Biochem 83:121-128, 2001

27. Meng W, Yonenobu K, Ariga K, et al: Localization of cathepsins $\mathrm{G}$ and $\mathrm{L}$ in spontaneous resorption of intervertebral discs in a rat experimental model. J Musculoskelet Neuronal Interact 2:171-176, 2001

28. Nerlich AG, Boos N, Wiest I, et al: Immunolocalization of major interstitial collagen types in human lumbar intervertebral discs of various ages. Virchows Arch 432:67-76, 1998

29. Nishida K, Gilbertson LG, Robbins PD, et al: Potential applications of gene therapy to the treatment of intervertebral disc disorders. Clin Orthop Relat Res 1 (Suppl 1):S234-S241, 2000

30. Nishimura K, Mochida J: Percutaneous reinsertion of the nucleus pulposus. An experimental study. Spine 23:1531-1539, 1998

31. Risbud M, Ringe J, Bhonde R, et al: In vitro expression of cartilage-specific markers by chondrocytes on a biocompatible hydrogel: implications for engineering cartilage tissue. Cell Transplant 10:755-763, 2001

32. Risbud MV, Albert TJ, Guttapalli A, et al: Differentiation of mesenchymal stem cells towards a nucleus pulposus-like phenotype in vitro: implications for cell-based transplantation therapy. Spine 29:2627-2632, 2004

33. Risbud MV, Shapiro IM, Vaccaro AR, et al: Stem cell regeneration of the nucleus pulposus. Spine J 4 (Suppl 6):348S-353S, 2004

34. Sakai D, Mochida J, Yamamoto Y, et al: Transplantation of mesenchymal stem cells embedded in Atelocollagen gel to the intervertebral disc: a potential therapeutic model for disc degeneration. Biomaterials 24:3531-3541, 2003

35. Seguin CA, Grynpas MD, Pilliar RM, et al: Tissue engineered nucleus pulposus tissue formed on a porous calcium polyphosphate substrate. Spine 29:1299-1307, 2004

36. Semenza GL, Roth PH, Fang HM, et al: Transcriptional regulation of genes encoding glycolytic enzymes by hypoxia-inducible factor 1. J Biol Chem 269:23757-23763, 1994

37. Silverman RP, Passaretti D, Huang W, et al: Injectable tissueengineered cartilage using a fibrin glue polymer. Plast Reconstr Surg 103:1809-1818, 1999

38. Stamm C, Westphal B, Kleine HD, et al: Autologous bone- marrow stem-cell transplantation for myocardial regeneration. Lancet 361:45-46, 2003

39. Steck E, Bertram H, Abel R, et al: Induction of intervertebral disc-like cells from adult mesenchymal stem cells. Stem Cells 23:403-411, 2005

40. Stern S, Lindenhayn K, Schultz O, et al: Cultivation of porcine cells from the nucleus pulposus in a fibrin/hyaluronic acid matrix. Acta Orthop Scand 71:496-502, 2000

41. Sztrolovics R, Alini M, Mort JS, et al: Age-related changes in fibromodulin and lumican in human intervertebral discs. Spine 24:1765-1771, 1999

42. Urban JP, Roberts S: Degeneration of the intervertebral disc. Arthritis Res Ther 5:120-130, 2003

43. Waddell G: Low back disability. A syndrome of Western civilization. Neurosurg Clin N Am 2:719-738, 1991

44. Waddell G: Low back pain: a twentieth century health care enigma. Spine 21:2820-2825, 1996

45. Wakitani S, Imoto K, Yamamoto T, et al: Human autologous culture expanded bone marrow mesenchymal cell transplantation for repair of cartilage defects in osteoarthritic knees. Osteoarthritis Cartilage 10:199-206, 2002

46. Walsh AJ, Bradford DS, Lotz JC: In vivo growth factor treatment of degenerated intervertebral discs. Spine 29:156-163, 2004

47. Yamamoto Y, Mochida J, Sakai D, et al: Upregulation of the viability of nucleus pulposus cells by bone marrow-derived stromal cells: significance of direct cell-to-cell contact in coculture system. Spine 29:1508-1514, 2004

48. Zhang YG, Guo X, Xu P, et al: Bone mesenchymal stem cells transplanted into rabbit intervertebral discs can increase proteoglycans. Clin Orthop Relat Res 430:219-226, 2005

Manuscript received July 15, 2005.

Accepted in final form August 15, 2005.

Address reprint requests to: Frank L. Acosta Jr., M.D., Department of Neurological Surgery, University of California, San Francisco, 505 Parnassus Avenue, M-779, Box 0112, San Francisco, California 94143-0112. email: acostaf@ neurosurg.ucsf.edu. 